\title{
COUPLED BEM AND FEM ANALYSIS OF FLUID-STRUCTURE INTERACTION IN DUAL COMPARTMENT TANKS
}

VASYL V. GNITKO, KYRYL G. DEGTYARIOV, VITALY V. NAUMENKO \& ELENA A. STRELNIKOVA*
A.N. Podgorny Institute for Mechanical Engineering Problems of the Ukrainian Academy of Sciences, Ukraine.

\begin{abstract}
The paper presents a fluid-structure interaction analysis of fuel tanks with cylindrical and spherical compartments partially filled with a liquid. The compound shell of revolution is considered as a container model. The shell is supposed to be thin, so the Kirchhoff-Love linear theory hypotheses are applied. The liquid is an ideal and incompressible one. Its properties and filling levels may be different within each compartment. The shell vibrations coupled with liquid sloshing under the force of gravity have been considered. The tank structure is modelled by a finite element method, whereas liquid sloshing in the compartments is described by a boundary element method. A system of singular integral equations is obtained for evaluating the fluid pressure. At the first stage, both spherical and cylindrical fluid-filled unconnected rigid shells are considered. Different filling levels as well as small radii of free surfaces are taken into account in problems of liquid sloshing in spherical shells. The sloshing frequencies in the presence of complete or partially covered free surfaces are determined for cylindrical shells. The boundary element method has proven to be effective and accurate in all the problems considered. At the second stage, the natural frequencies and modes of the dual compartment tank are obtained including sloshing, elasticity, and gravity effects.

Keywords: baffles, boundary and finite element methods, fluid-structure interaction, free vibrations, fuel tanks, sloshing
\end{abstract}

\section{INTRODUCTION}

The low frequency oscillations of free surfaces in partially filled containers significantly increase dynamic responses of structures containing liquids. This phenomenon is known as sloshing. It can result in severe structural damage, loss of stability, and failure.

In order to suppress sloshing, a variety of methods have been proposed, simulated, and tested. The boundary element method (BEM) has been successfully used in solution of both linear and non-linear sloshing problems. In order to solve the large-scale problems, researchers developed the multi-domain boundary element method (MBEM). The multi-domain collocation strategy was briefly introduced by Brebbia et al. [1] and then developed by Wang and Gao [2]. The MBEM is especially effective if the computational domain has a complicated structure. It is successfully applied for numerical simulation of sloshing in multi-compartment fuel tanks with different types of baffles.

The effect of baffles on sloshing frequencies was studied by Biswal et al. [3]. The numerical method using a finite element formulation was developed by Kumar and Sinhamahapatra [4] to analyze dynamic effects of perforated vertical baffles. Sloshing in spherical tanks for liquefied natural gas carriers was studied by Faltinsen and Timokha [5] and for water supply towers by Curadelli et al. [6]. Ravnik et al. [7] presented a fluid-structure interaction analysis of the cylindrical fuel tank with two compartments partially filled with a liquid. But fluid-structure interaction problems for fuel tanks of more complicated shapes are still not fully described in the literature. 


\section{PROBLEM STATEMENT}

In this paper, we describe applications of multi-domain and single-domain boundary element methods to the fluid-structure interaction analysis of compound fuel tanks.

Consider a fuel tank with cylindrical and spherical compartments. The internal baffles can be installed in both compartments for slosh suppression. The structure and its sketch are shown in Fig. 1.

Assume that both compartments are partly filled with an incompressible ideal liquid. Fluid motion is considered to be irrotational.

Suppose $\sigma$ is a wetted part of the structure surfaces, $S_{01}$ and $S_{02}$ are free surfaces of the liquid in spherical and cylindrical compartments, respectively. Let $S_{0}$ be $S_{0}=S_{01} \cup S_{02}$. The wetted surface $\sigma$ consists of four parts, namely, $\sigma=S_{w 1} \cup S_{w 2} \cup S_{\text {bot }} \cup S_{\text {baf }}$. Here $S_{w 1}$ and $S_{w 2}$ are the wetted surfaces of cylindrical and spherical parts, $S_{\text {bot }}$ is a bottom surface of the tank and $S_{\text {baf }}$ is a baffle surface. The domains occupied with the liquid are denoted by $\Sigma_{1}$ and $\Sigma_{2}$ for cylindrical and spherical compartments, respectively. Let $\Sigma_{12}=\partial \Sigma_{1} \cap \partial \Sigma_{2}$ be the common part of wetted surface areas. The liquid densities are $\rho_{1}$ and $\rho_{2}$, and filling levels are $h_{1}$ and $h_{2}$ in $\Sigma_{1}$ and $\Sigma_{2}$, respectively. Let $R_{i r}$ be the inner radius of the ring free surface (Fig. 1).

Consider at first free vibrations of the empty elastic shell structure. Assume that a time dependent vector of the shell displacements $\tilde{\mathbf{U}}$ is given by

$$
\tilde{\mathbf{U}}=\tilde{\mathbf{u}} \exp (i \Omega t) ; \quad \tilde{\mathbf{u}}=\left(u_{1}, u_{2}, u_{3}\right),
$$

where $\Omega$ is a vibration frequency. The time factor $\exp (i \Omega)$ will be omitted further on. After separation of the time factor, the shell vibrations are described by the system of three partial differential equations

$$
\sum_{i=1}^{3} L_{i j} u_{i}=\Omega^{2} u_{j}, \quad j=1,2,3
$$

where $L_{i j}$ are linear differential operators of the Kirchhoff-Love shell theory described by Wan et al. [8]. To obtain the natural frequencies $\Omega_{k}$ and modes $\mathbf{u}_{k}(k=\overline{1, N})$ of the shell structure, the finite element method is applied [7].
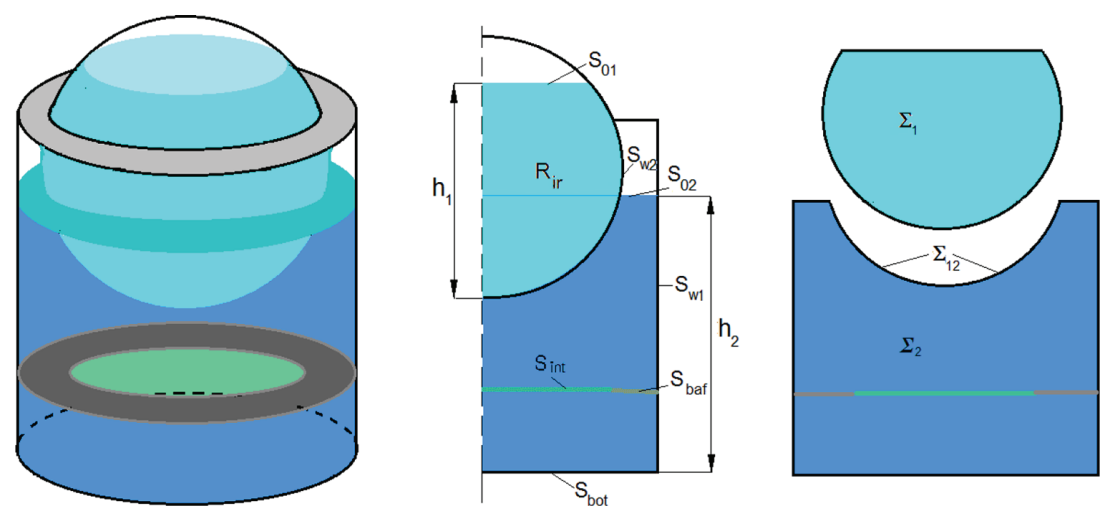

Figure 1: Shell structure with an internal baffle, its sketch and fluid subdomains. 
Let $\mathbf{U}=\left(U_{1}, U_{2}, U_{3}\right)$ be displacements of the fluid-filled elastic shell structure. After forming the global stiffness and mass matrices $\mathbf{L}$ and $\mathbf{M}$, the following equation of motion for the tank filled with the liquid has been obtained [7]:

$$
\mathbf{L U}+\mathbf{M} \ddot{\mathbf{U}}=\mathbf{P} .
$$

Here $\mathbf{P}$ gives the fluid dynamical pressure onto the shell structure normal to its surface.

Assuming the flow to be inviscid and irrotational, the incompressible fluid motion in the $3 \mathrm{D}$ tank is described by the Laplace equation

$$
\nabla^{2} \Phi=0
$$

where $\Phi$ is a velocity potential.

To determine this potential a mixed boundary value problem for the Laplace equation is formulated in the double domain $\Sigma_{1} \cup \Sigma_{2}$. The non-penetration condition on the wetted tank surfaces $\sigma$ is following:

$$
\left.\frac{\partial \Phi}{\partial \mathbf{n}}\right|_{\sigma}=\frac{\partial w}{\partial t}
$$

Here $\mathbf{n}$ is an external unit normal to the tank wetted surfaces, $w$ denotes a normal component of the displacement vector $\mathbf{U}$, namely, $w=(\mathbf{U}, \mathbf{n})$. Let functions $\varsigma_{1}(t, x, y)$ and $\varsigma_{2}(t, x, y)$ be free surface elevations in the first and second compartments (Fig. 1). By $p_{i}$ denote the pressure on the free surfaces $S_{0 i}(i=1,2)$. Let $p_{0}$ be an atmospheric pressure. Then the kinematic and dynamic boundary conditions on $S_{01}$ and $S_{02}$ can be expressed as follows:

$$
\left.\frac{\partial \Phi}{\partial \mathbf{n}}\right|_{S_{0 i}}=\frac{\partial \zeta_{i}}{\partial t} ; \quad p_{i}-\left.p_{0}\right|_{S_{0 i}}=0 ; \quad i=1,2
$$

On the free surfaces, the following formulae for $p_{i}(i=1,2)$ are valid:

$$
p_{1}-p_{0}=-\left.\rho_{1}\left(\frac{\partial \Phi}{\partial t}+g \zeta_{1}\right)\right|_{S_{01}} ; \quad p_{2}-p_{0}=-\left.\rho_{2}\left(\frac{\partial \Phi}{\partial t}+g \zeta_{2}\right)\right|_{S_{02}} .
$$

Here $\mathrm{g}$ is the gravity acceleration.

Denote by $\Phi^{(i)}$ values of the velocity potential $\Phi$ at points $P \in \partial \Sigma_{i}(i=1,2)$.

Equations (1) and (2) are solved simultaneously using the shell fixation conditions relative to $\mathbf{U}$, boundary conditions (3) and (4), and the following expressions for dynamical components of the liquid pressure on elastic walls:

$$
p_{l}=(\mathbf{P}, \mathbf{n})=\left\{\begin{array}{c}
-\rho_{1} \frac{\partial \Phi^{(1)}}{\partial t} ; \quad P \in S_{w 1} \cup S_{\mathrm{bot}} \\
-\rho_{2} \frac{\partial \Phi^{(2)}}{\partial t} ; \quad P \in S_{w 2} \backslash \Sigma_{12} \\
-\left(\rho_{1} \frac{\partial \Phi^{(1)}}{\partial t}-\rho_{2} \frac{\partial \Phi^{(2)}}{\partial t}\right) ; P \in \Sigma_{12} .
\end{array}\right.
$$


To define modes of shell vibrations coupled with liquid sloshing, we will represent displacements of the fluid-filled tank as $\mathbf{U}=\mathbf{u}_{\mathrm{f}} \times \exp (i \omega t)$. Here $\omega$ and $\mathbf{u}_{\mathbf{f}}$ are natural frequencies and vibration modes of the fluid-filled shell structure.

\section{THE MODE SUPERPOSITION METHOD FOR COUPLED DYNAMIC PROBLEMS}

Consider the vibration modes of the fluid-filled tank in a form

$$
\mathbf{U}=\sum_{k=1}^{N} c_{k} \mathbf{u}_{k}
$$

where $c_{k}=c_{k}(t)$ are unknown coefficients, and $\mathbf{u}_{k}$ are eigenmodes of the empty tank. In other words, the mode of vibration of the fluid-filled tank is determined as a linear combination of eigenmodes of the empty shell structure. Note that the following relationships are fulfilled [7]

$$
\mathbf{L}\left(\mathbf{u}_{k}\right)=\Omega_{k}^{2} \mathbf{M}\left(\mathbf{u}_{k}\right), \quad\left(\mathbf{M}\left(\mathbf{u}_{k}\right), \mathbf{u}_{j}\right)=\delta_{k j} .
$$

Hence

$$
\left(\mathbf{L}\left(\mathbf{u}_{k}\right), \mathbf{u}_{j}\right)=\Omega_{k}^{2} \delta_{k j}
$$

where $\Omega_{k}$ is the $k$-th frequency of the empty tank vibrations. Equations (6) and (7) show that the abovementioned vibration modes have to be orthonormalized with respect to the mass matrix.

Let also introduce $\mathbf{u}_{k}^{i}$ as follows:

$$
\mathbf{u}_{k}= \begin{cases}\mathbf{u}_{k}^{1} ; & P \in \Sigma_{1}, \\ \mathbf{u}_{k}^{2} ; & P \in \Sigma_{2} .\end{cases}
$$

Consider $\Phi$ as a sum of two potentials $\Phi=\Phi_{1}+\Phi_{2}$ as it was done by Degtyarev et al. [9]. Represent potential $\Phi_{1}$ as the following series:

$$
\Phi_{1}=\sum_{k=1}^{N} \dot{c}_{k}(t) \phi_{1 k}
$$

Here the time-dependant coefficients $c_{k}(t)$ are defined in eqn (5). To determine functions $\varphi_{1 k}$ $(k=\overline{1, N})$, we have the following boundary value problems:

$$
\nabla^{2} \phi_{1 k}^{i}=0 ; \quad P \in \Sigma_{i} ;\left.\frac{\partial \phi_{1 k}^{i}}{\partial \mathbf{n}}\right|_{S_{w i}}=w_{k}^{i} ;\left.\frac{\partial \phi_{1 k}^{1}}{\partial \mathbf{n}}\right|_{S_{\mathrm{bot}}}=w_{k}^{i} ;\left.\phi_{1 k}^{i}\right|_{S_{i 0}}=0 .
$$

Here, $w_{k}^{1}, w_{k}^{2}$ are normal components of the mode $\mathbf{u}_{k}$ in the first and second compartments, $w_{k}^{i}=\left(\mathbf{u}_{k}^{i}, \mathbf{n}\right)$. So functions $\phi_{1 k}^{1}$ and $\phi_{1 k}^{2}$ are solutions of problems (9) for $i=1$ and $i=2$, accordingly, as in Ref. [10].

To determine potential $\Phi_{2}$, we must solve the boundary value problems of fluid vibrations in two disconnected compartments with rigid walls. These problems are solved separately for spherical and cylindrical parts. To obtain the sloshing modes, the following sequence of boundary value problems for auxiliary functions $\psi_{2 k}^{i}(i=1,2)$ is formulated: 


$$
\begin{gathered}
\Delta \psi_{2 k}^{i}=0 ;\left.\quad \frac{\partial \psi_{2 k}^{i}}{\partial \mathbf{n}}\right|_{S_{w i}}=0 ;\left.\frac{\partial \psi_{2 k}^{1}}{\partial \mathbf{n}}\right|_{S_{\mathrm{bot}}}=0 ; \\
\left.\frac{\partial \psi_{2 k}^{i}}{\partial \mathbf{n}}\right|_{S_{0 i}}=\frac{\partial S_{i}}{\partial t} ; \quad \frac{\partial \psi_{2 k}^{i}}{\partial t}+\left.g \zeta_{i}\right|_{S_{0 i}}=0, k=\overline{1, N} ; \quad i=1,2 .
\end{gathered}
$$

Suppose hereinafter $\psi_{2 k}(t, x, y, z)=e^{i \chi_{k} t} \phi_{2 k}(x, y, z)$. Then we obtain the following eigenvalue problems $[9,10]$ for each $\phi_{2 k}^{i}(i=1,2)$ in the liquid domains $\Sigma_{i}(i=1,2)$ :

$$
\Delta \phi_{2 k}^{i}=\left.0\right|_{\Sigma_{i}} ;\left.\frac{\partial \phi_{2 k}^{i}}{\partial \mathbf{n}}\right|_{S_{w i}}=0 ;\left.\frac{\partial \phi_{2 k}^{1}}{\partial \mathbf{n}}\right|_{S_{\mathrm{bot}}}=0 ; \frac{\partial \phi_{2 k}^{i}}{\partial \mathbf{n}}=\left.\frac{\chi_{k}^{2}}{g} \phi_{2 k}^{i}\right|_{S_{0 i}} ; \int_{S_{0 i}} \phi_{2 k}^{i} d S=0 .
$$

So for potentials $\Phi_{1}$ and $\Phi_{2}$ we obtain the next representations:

$$
\Phi_{1}=\left\{\begin{array}{ll}
\sum_{k=1}^{M_{1}} A \phi_{1 k}^{1}, & P \in \Sigma_{1}, \\
\sum_{k=1}^{M_{2}} A_{k} \phi_{1 k}^{2}, & P \in \Sigma_{2},
\end{array} ; \Phi_{2}= \begin{cases}\sum_{k=1}^{L_{1}} \dot{d}_{k} \phi_{2 k}^{1}, & P \in \Sigma_{1}, \\
\sum_{k=1}^{L_{2}} \dot{d}_{k} \phi_{2 k}^{2}, & P \in \Sigma_{2} .\end{cases}\right.
$$

Here $c_{k}(t), d_{k}(t)$ are unknown time-dependant coefficients.

The effective numerical procedure for solution of eigenvalue problems (10) using the single and multi-domain boundary element methods has been introduced in Refs. [7, 10].

Thus, the problem under consideration involves the following steps. First, it is necessary to obtain the sloshing frequencies and modes $\phi_{2 k}^{i}$ using rigid wall assumption. Second, we obtain the natural frequencies $\Omega_{k}$ and modes $\mathbf{u}_{k}$ of the empty elastic tank. Then we define the free vibration frequencies and modes $\phi_{1 k}^{i}$ of the elastic tank without considering effects of sloshing. When functions $\phi_{1 k}^{i}$ and $\phi_{2 k}^{i}$ are defined, we substitute them in eqns (11) and (1) and obtain the system of ordinary differential equations as it was done in Ref. [10]. Finally, the flow-induced vibrations of elastic structures are studied.

To define functions $\phi_{1 k}^{i}$ and $\phi_{2 k}^{i}$ we use the boundary element method in its direct formulation [1]. Dropping indices $i, 1 k$ and $2 k$ one can obtain the main integral equation in the following form:

$$
2 \pi \phi\left(P_{0}\right)=\iint_{S} q \frac{1}{\left|P-P_{0}\right|} d S-\iint_{S} \phi \frac{\partial}{\partial \mathbf{n}} \frac{1}{\left|P-P_{0}\right|} d S .
$$

Here, $S=\sigma \cup S_{0}$, points $P$ and $P_{0}$ belong to the surface $\mathrm{S}$. The value $\left|P-P_{0}\right|$ represents Cartesian distance between the points $P$ and $P_{0}$. In doing so, the function $\varphi$ defined on the wetted tank surface $\sigma$ presents the pressure, and the function $q$ defined on the free surface $S_{0}$, is the flux, $q=\partial \phi / \partial \mathbf{n}$. To apply the MBEM, we introduce the artificial interface surface $S_{\text {int }}[11]$ [12]. In MBEM, the computational domain is divided into a number of subdomains, and the BEM algebraic equations are established for each subdomain. Then the global system of algebraic equations is formed by assembling results of all subdomains in terms of the equilibrium and matching conditions over common interface nodes. This system has a blocked and sparse character. 
The basic procedure is to start with the standard boundary integral equation for potential (12), replace Cartesian coordinates $(x, y, z)$ with cylindrical ones $(r, \theta, z)$, and integrate with respect to $z$ and $\theta$. We use furthermore the cylindrical coordinate system, and represent unknown functions as Fourier series by the circumferential coordinate $\theta$

$w_{k}^{i}(r, z, \theta)=w_{k}^{i}(r, z) \cos \alpha \theta ; \quad \phi_{j k}^{i}(r, z, \theta)=\phi_{j k}^{i}(r, z) \cos \alpha \theta ; i=1,2 ; j=1,2 ; k=1,2, \ldots$,

where $\alpha$ is a given integer (the number of nodal diameters). In this case, the solution is independent of the angular coordinate $\theta$, and the three-dimensional problem is reduced to a two-dimensional one in the radial coordinate $r$ and the axial coordinate $z$.

Let $\Gamma$ be a generator of the surface $\sigma$. Using (12), (13) we have obtained the following system of singular integral equations for unknown functions $\varphi$ and $q$ in problem (9):

$$
\begin{gathered}
2 \pi \phi\left(z_{0}\right)+\int_{\Gamma} \phi(z) Q\left(z, z_{0}\right) r(z) d \Gamma-\int_{0}^{R} q(\rho) \Psi\left(P, P_{0}\right) \rho d \rho=\int_{\Gamma} w(z) \Psi\left(P, P_{0}\right) r(z) d \Gamma_{1} ; P_{0} \in \sigma ; \\
\int_{\Gamma} \phi(z) Q\left(z, z_{0}\right) r(z) d \Gamma-\int_{0}^{R} q(\rho) \Psi\left(P, P_{0}\right) \rho d \rho=\int_{\Gamma} w(z) \Psi\left(P, P_{0}\right) r(z) d \Gamma_{1} ; P_{0} \in S_{0} .
\end{gathered}
$$

Here

$$
\begin{aligned}
& Q\left(z, z_{0}\right)=\frac{4}{\sqrt{a+b}}\left\{\frac{1}{2 r}\left[\frac{r^{2}-r_{0}^{2}+\left(z_{0}-z\right)^{2}}{a-b} \mathrm{E}_{\alpha}(k)-\mathrm{F}_{\alpha}(k)\right] n_{r}+\frac{z_{0}-z}{a-b} \mathrm{E}_{\alpha}(k) n_{z}\right\} ; \\
& \Psi\left(P, P_{0}\right)=\frac{4}{\sqrt{a+b}} \mathrm{~F}_{\alpha}(k) ; \mathrm{E}_{\alpha}(k)=(-1)^{\alpha}\left(1-4 \alpha^{2}\right) \int_{0}^{\pi / 2} \cos 2 \alpha \psi \sqrt{1-k^{2} \sin ^{2} \psi} d \psi ; \\
& \mathrm{F}_{\alpha}(k)=(-1)^{\alpha} \int_{0}^{\pi / 2} \frac{\cos 2 \alpha \psi d \psi}{\sqrt{1-k^{2} \sin ^{2} \psi}} ; a=\rho^{2}+\rho_{0}^{2}+\left(z-z_{0}\right)^{2} ; \quad b=2 \rho \rho_{0} ; k^{2}=\frac{2 b}{a+b} .
\end{aligned}
$$

The system of singular integral equations for mixed boundary value problem (10) has been obtained in Ref. [10]. Numerical procedures to solve these problems based on the boundary element method are described in details in the papers $[7,9,11]$.

\section{SOME NUMERICAL RESULTS}

\subsection{Low frequency sloshing modes for partially filled spherical shells}

\subsubsection{Spherical shells without baffles}

Now we turn to the problem of sloshing in the spherical container without baffles. Radius of the sphere is $R_{1}=1 \mathrm{~m}$; the filling level is $h_{1}$.

The numerical simulation has been provided for different values of $h_{1}\left(0.2<h_{1} / R_{1}<1.99\right)$ and different modes $\alpha(\alpha=\overline{0,3})$. Both SBEM and MBEM are applied here. The boundary elements with constant approximation of unknowns inside elements are used. In SBEM there are 200 elements along the spherical surface and 150 elements along the free surface. In MBEM we divide the computational domain into two parts by an artificial interface surface at $h_{\text {int }}=0.5 h_{1}$ using 100 boundary elements in each subdomain along the spherical surface and 150 elements along the free surface. 
We use practically the same mesh to find a numerical approximation of low eigenvalues for the so-called 'ice-fishing problem'. In this problem, formally, we should consider an infinitely wide and deep ocean covered with ice, with a small round fishing hole. Sloshing in such 'containers' was studied by McIver [12]. We approximate this infinite case using the spherical tank with the small round hole on its top. It allows us to compare our numerical results with those obtained in the papers [12-14].

Proposed boundary element approach gives nearly the same values as the other authors. In Tables 1 and 2 we compare our results obtained by using SBEM and MBEM with those obtained in Refs. [12-14] for axisymmetric $(\alpha=0)$ and non-axisymmetric $(\alpha=1)$ modes. Four first frequencies $(m=\overline{1,4})$ are evaluated for each $a$. Here we consider different filling levels $h_{1}$. The value $h_{1} / R_{1}=1.99$ corresponds to the ice-fishing problem. The results of Faltinsen and Timokha [13], and Kulczycki et al. [14] are very close. Both Refs. [13-14] and [12] results analytically approximate the sloshing eigenvalues of the spherical tank.

It should be noted that results obtained by SBEM are more precise than MBEM ones, but the matrix size in SBEM is twice larger compared with MBEM. If un-baffled tanks are at low filling levels, it is preferable to use SBEM. The numerical analysis demonstrates that the lowest liquid sloshing frequency occurs for mode $a=1$.

Considering our approximate natural sloshing modes one can observe how free surface profiles change with the liquid depth. These results are illustrated in Fig. 2 for the three lowest eigenvalues of the mode $a=1$. Here numbers $1,2,3,4$ correspond to the different non-dimensional filling levels: $h_{1} / R_{1}=1.0 ; 0.2 ; 1.8 ; 1.9$, respectively.

In the spherical tank with $0<h_{1} / R_{1}<0.5$ the lowest mode presents a spatial wave pattern that look like inclination of an almost flat free surface. Increasing the liquid depth yields more

Table 1: Axisymmetric slosh frequencies of the fluid-filled spherical shell, Hz.

\begin{tabular}{llrrrrr}
\hline & & \multicolumn{4}{c}{ Filling level $h_{1}, \mathrm{~m}$} \\
\cline { 3 - 7 }$m$ & Method & $h_{1}=0.2$ & $h_{1}=0.6$ & $h_{1}=1.0$ & $h_{1}=1.8$ & $h_{1}=1.99$ \\
\hline 1 & {$[13,14]$,} & 3.8261 & 3.6501 & 3.7451 & 6.7641 & 29.0500 \\
& {$[12]$} & 3.8261 & 3.6501 & 3.7451 & 6.7641 & 29.2151 \\
& MBEM & 3.4034 & 3.5455 & 3.7294 & 6.6098 & 30.7081 \\
& SBEM & 3.8314 & 3.6510 & 3.7456 & 6.7665 & 29.1811 \\
2 & [13,14], & 9.2561 & 7.2659 & 6.9763 & 12.1139 & 51.8122 \\
& [12] & 9.2561 & 7.2659 & 6.9763 & 12.1139 & 52.0467 \\
& MBEM & 9.2636 & 7.2893 & 6.9796 & 12.0008 & 52.9393 \\
& SBEM & 9.2686 & 7.2684 & 6.9780 & 12.1205 & 52.0255 \\
3 & [13,14], & 14.7556 & 10.7443 & 10.1474 & 17.3960 & 74.2909 \\
& {$[12]$} & 14.7556 & 10.7443 & 10.1474 & 17.3960 & 74.5537 \\
& MBEM & 14.9214 & 10.7483 & 10.1496 & 17.3136 & 75.3139 \\
& SBEM & 14.7763 & 10.7502 & 10.1512 & 17.4086 & 74.5547 \\
4 & {$[13,14]$,} & 20.1187 & 14.1964 & 13.3041 & 22.6579 & 96.6207 \\
& {$[12]$} & 20.1187 & 14.1964 & 13.3041 & 22.6570 & 96.9560 \\
& MBEM & 20.2066 & 14.2023 & 13.3083 & 22.5962 & 97.7771 \\
& SBEM & 20.1498 & 14.2056 & 13.3110 & 22.6777 & 96.9021 \\
\hline
\end{tabular}


Table 2: Non-axisymmetric slosh frequencies of the fluid-filled spherical shell, Hz.

\begin{tabular}{rlrrrrr}
\hline & & \multicolumn{4}{c}{ Filling level $h_{1}, \mathrm{~m}$} \\
\cline { 3 - 7 }$m$ & Method & $h_{1}=0.2$ & $h_{1}=0.6$ & $h_{1}=1.0$ & $h_{1}=1.8$ & $h_{1}=1.99$ \\
\hline 1 & {$[13,14]$,} & 1.0723 & 1.2625 & 1.5601 & 3.9593 & 18.9838 \\
& {$[12]$} & 1.0723 & 1.2625 & 1.5601 & 3.9593 & 19.1582 \\
& MBEM & 1.1034 & 1.2777 & 1.5638 & 3.9606 & 19.1603 \\
& SBEM & 1.0723 & 1.2626 & 1.5603 & 3.9508 & 19.1130 \\
2 & {$[13,14]$,} & 6.2008 & 5.3860 & 5.2755 & 9.4534 & 41.3491 \\
& {$[12]$} & 6.2008 & 5.3860 & 5.2755 & 9.4534 & 41.7683 \\
& MBEM & 6.1227 & 5.3534 & 5.2749 & 9.4582 & 41.5327 \\
& SBEM & 6.2090 & 5.3697 & 5.2764 & 9.4538 & 41.5333 \\
& {$[13,14]$,} & 11.8821 & 8.9418 & 8.5044 & 14.7548 & 63.5354 \\
& {$[12]$} & 11.8821 & 8.9418 & 8.5044 & 14.7548 & 64.0323 \\
& MBEM & 11.9650 & 8.9529 & 8.5062 & 14.7648 & 63.9483 \\
& SBEM & 11.8981 & 8.9429 & 8.5069 & 14.7574 & 63.8783 \\
4 & {$[13,14]$,} & 17.3581 & 12.4234 & 11.6835 & 20.0224 & 85.9166 \\
& {$[12]$} & 17.3584 & 12.4234 & 11.6835 & 20.0224 & 86.3001 \\
& MBEM & 17.4540 & 12.4276 & 11.6863 & 20.0394 & 86.2972 \\
& SBEM & 17.3842 & 12.4291 & 11.6884 & 20.0278 & 86.2034 \\
\hline
\end{tabular}

complicated free surface profiles. Figure 3 demonstrates the spatial wave patterns for $a=1$, $m=1,2,3$ at $h_{1} / R_{1}=1.8$.

\subsubsection{Spherical shells with baffles}

Consider the rigid spherical tank of radius $R_{1}=1 \mathrm{~m}$ filled to the depth $h_{1}=1.4 \mathrm{~m}$. The inner periphery of the tank contains a thin rigid-ring baffle. The baffle position is $h_{\mathrm{baf}}=1 \mathrm{~m}$. The different annular orifices in the baffle are considered. Radii of these orifices are radii $R_{\text {int }}$ of the interface surfaces. The first four frequencies for mode $\alpha=1$ are evaluated for radii $R_{\text {int }}=1.0 \mathrm{~m}$, $R_{\text {int }}=0.7 \mathrm{~m}$, and $R_{\text {int }}=0.2 \mathrm{~m}$. Note that $R_{\text {int }}=1.0 \mathrm{~m}$ correspond to the un-baffled tank. The frequencies are presented in Table 3.

The results show that frequencies decrease as radius of the baffle orifice is decreased. Only the lowest eigenvalue is essentially influenced by installing the baffles.

Figure 4 demonstrates the first non-axisymmetric modes of liquid vibrations in spherical tanks with and without baffles. When the baffle is installed, the mode shape becomes almost flat.

\subsection{Sloshing frequencies for partially filled cylindrical tanks with covered free surfaces}

Consider eigenvalue problem (10) for the second fluid domain $\Sigma_{2}$ bounded by cylindrical and spherical surfaces (Fig. 1). By $H$ denote a height of the cylinder, by $R_{1}, R_{2}$ radii of the spherical and cylindrical parts, and by $h_{2}$ the filling level in $\Sigma_{2}$. Let $\left(0, z_{s}\right)$ be centre coordinates of the sphere. The shell structure has following dimensions: $R_{1}=1 \mathrm{~m} ; R_{2}=1.2 \mathrm{~m} ; z_{s}=1.5 \mathrm{~m} ; H$ $=2 . \mathrm{m}$. The modes and frequencies are evaluated for different $h_{2}$ in the range of 


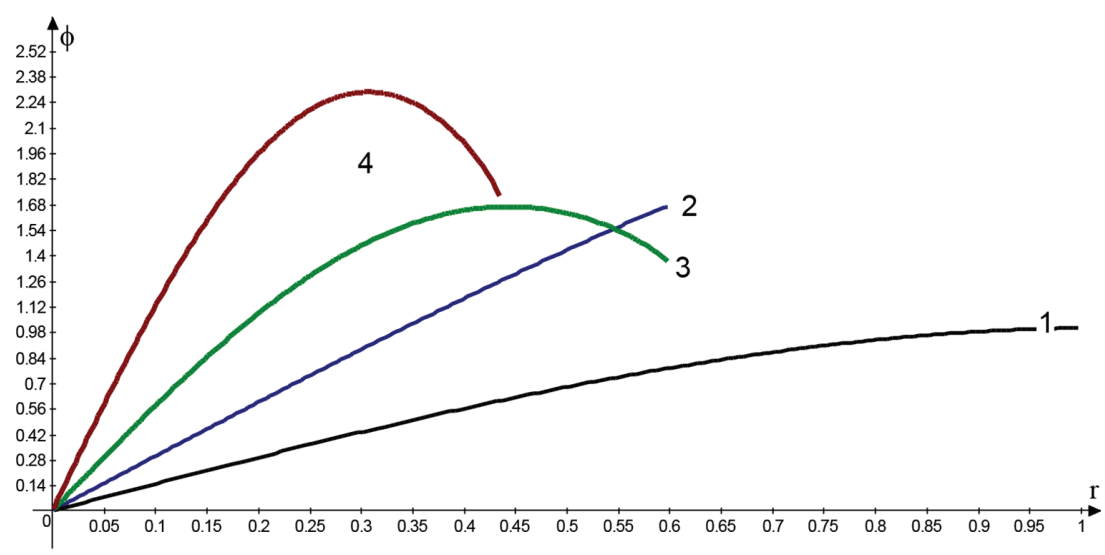

a) $\alpha=1, m=1$

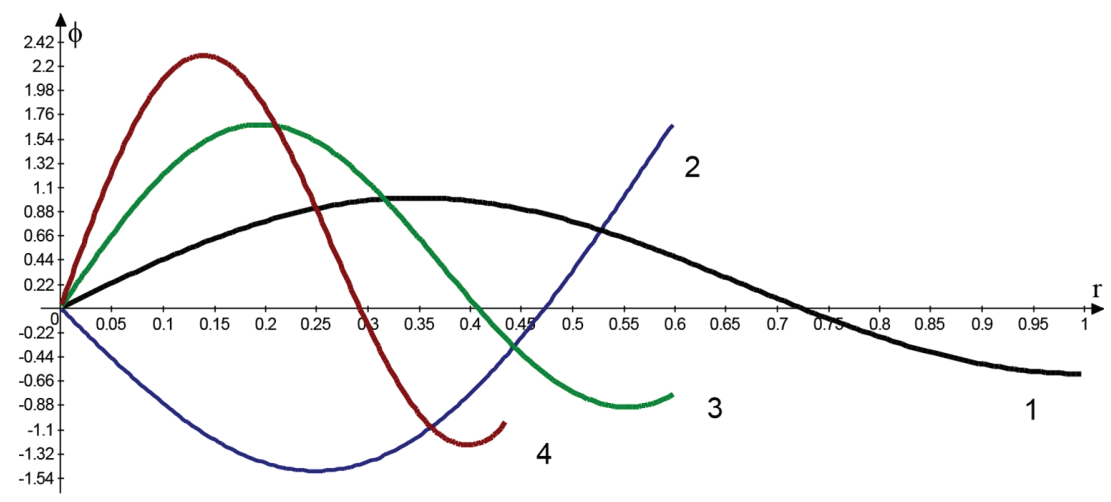

b) $\alpha=1, m=2$

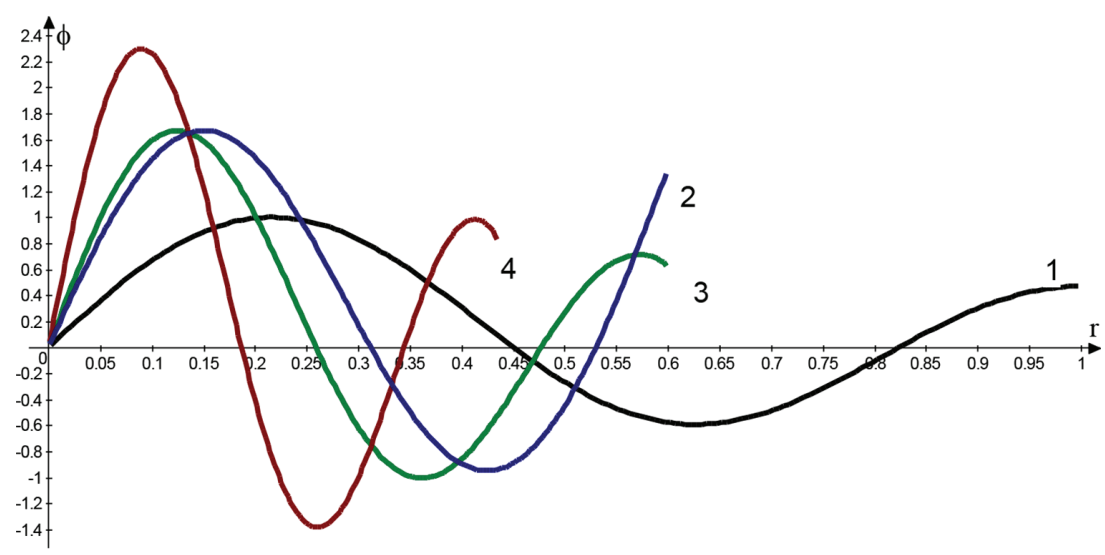

c) $\quad \alpha=1 . m=3$

Figure 2: The radial wave profiles $m=1,2,3$ for different non-dimensional liquid depths $h_{1} / R_{1}$. 

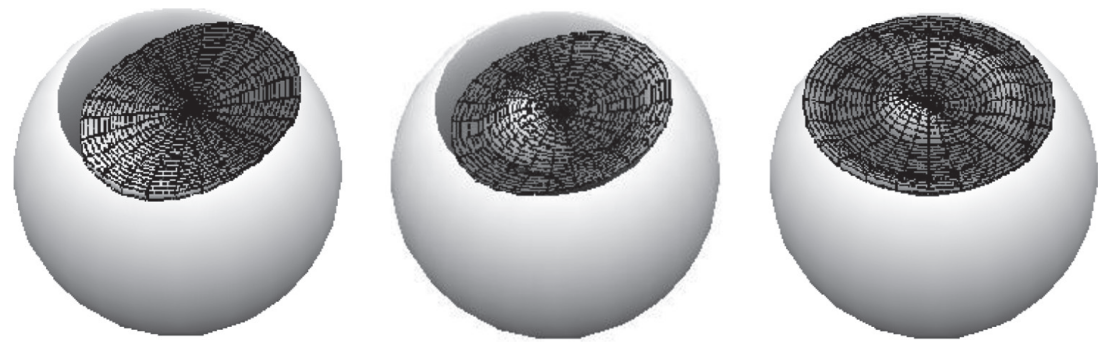

Figure 3: Spatial wave patterns for $\alpha=1 ; m=1,2,3$.

Table 3: Sloshing frequencies of the fluid-filled spherical shell with baffles, Hz.

\begin{tabular}{llll}
\hline & \multicolumn{3}{c}{$\omega^{2} / \mathrm{g}$} \\
\cline { 2 - 4 }$m$ & $R_{\text {int }}=1.0 \mathrm{~m}$ & $R_{\text {int }}=0.7 \mathrm{~m}$ & $R_{\text {int }}=0.2 \mathrm{~m}$ \\
\hline 1 & 2.1232 & 2.0435 & 1.4234 \\
2 & 5.9800 & 5.9723 & 5.8405 \\
3 & 9.4789 & 9.4785 & 9.4567 \\
4 & 12.9431 & 12.9430 & 12.9358 \\
\hline
\end{tabular}
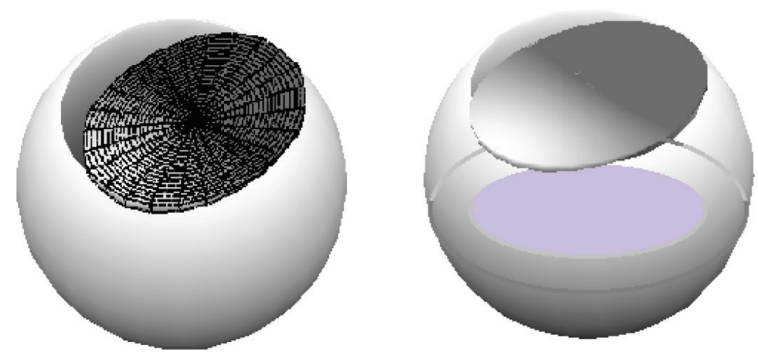

Figure 4: Spatial mode shapes for $a=1 ; m=1$ of un-baffled a) and baffled tanks b).

$0.5<h_{2} / R_{1}<2.39$. The inner radius $R_{i r}$ of the ring free surface is defined by the dive level of the spherical shell $z_{s}$ and the filling level $h_{2}$ as follows: $R_{i r}=\sqrt{R_{1}^{2}-\left(h_{2}-z_{s}\right)^{2}}$.

The results are illustrated in Fig. 5 for two lowest eigenvalues of the mode $\alpha=1$. Here numbers 1,2 correspond to frequencies of cylindrical tanks with ring and circular free surfaces, respectively. If $h_{2}=0.5 \mathrm{~m}$, then both tanks have the complete circular free surfaces.

Figure 5a) and b) demonstrates changes in the first and second frequencies of the mode $a$ $=1$ via the filling level $h_{2}$. The values of frequencies increase significantly with increasing the inner radius of the ring free surface. The maximum values of frequencies correspond to $h_{2}=z_{s} ; \quad R_{i r}=R_{1}$. Here we get the analogy with the "ice-fishing problem" for the spherical shell with $h_{1} / R_{1}=1.99$ (see Tables 1 and 2). Sloshing frequencies of cylindrical shell with circular free surface are stabilized when $h_{2}>\mathrm{z}_{\mathrm{s}}$; one can observe here the typical monotonic 


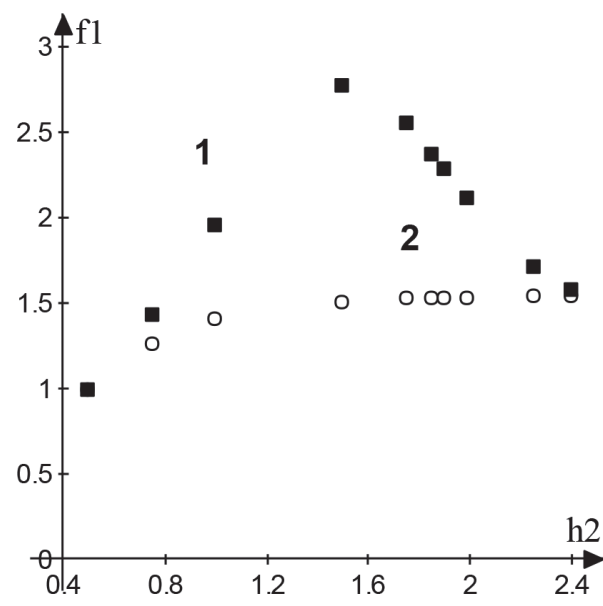

a)

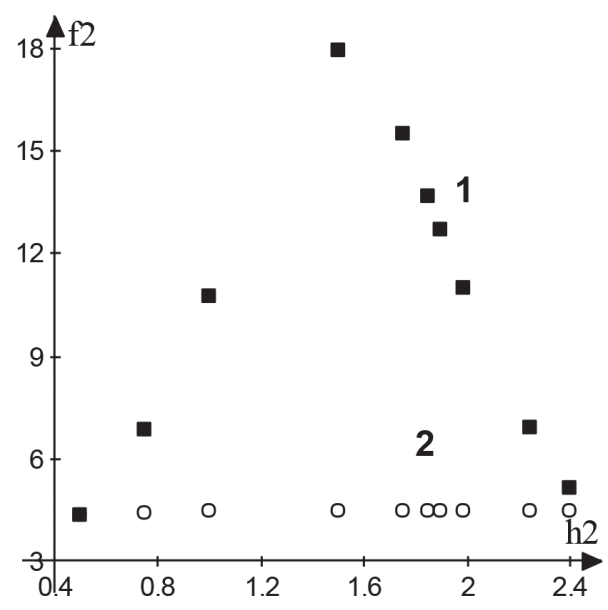

b)

Figure 5: Frequencies of cylindrical shell with ring and circular free surfaces.

dependences. The benefit of a partly covered free surface is that it increases the natural frequencies.

\subsection{Modes and frequencies of the empty cylindrical-spherical tank}

Consider the elastic spherical-cylindrical tank without baffles (Fig. 1). The shell structure is clamped at the bottom edge. Natural frequencies $\Omega_{k}$ and modes $\mathbf{u}_{k}, k=1, N$ of the shell structure without the liquid are calculated using FEM as it is proposed in Ref. [7]. The shell structure has the following dimensions: $R_{1}=1 \mathrm{~m}, R_{2}=1.2 \mathrm{~m}, z_{s}=1.5 \mathrm{~m}, H=2.0 \mathrm{~m}$, wall thickness $h=0.025 \mathrm{~m}$; and material properties: Young's modulus $E=2 \cdot 10^{5} \mathrm{MPa}$, Poisson's ratio $v=0.3$, density $\rho_{s}=2700 \mathrm{~kg} / \mathrm{m}^{3}$. Four modes of vibration are shown in Fig. 6 .

The modes 6a) and $6 \mathrm{~b}$ ) correspond to the duplicate frequency $\Omega_{1}=\Omega_{2}=4.6359 \mathrm{~Hz}$. This frequency is the lowest one of the mode $\alpha=1$. The axisymmetric mode $6 \mathrm{c}$ ) corresponds to the frequency $\Omega_{3}=5.9312 \mathrm{~Hz}$, that is the lowest one of the mode $\alpha=0$. The axisymmetric mode $6 \mathrm{~d}$ ) corresponds to the frequency $\Omega_{4}=50.9721 \mathrm{~Hz}$.

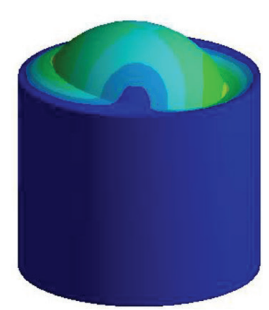

a)

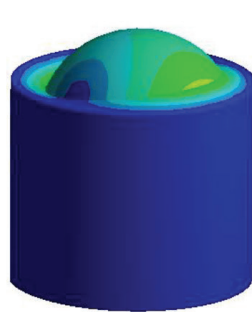

b)

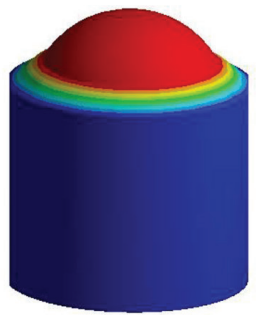

c)

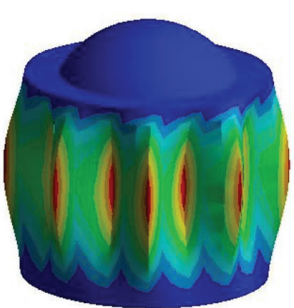

d)

Figure 6: Modes of the empty cylindrical-spherical tank. 
Table 4: Frequencies of the fluid-filled double tank, Hz.

\begin{tabular}{llll}
\hline$m$ & $\omega$ & Dominant vibration & $a$ \\
\hline 1 & 0.6223 & Sphere sloshing & 1 \\
2,3 & 0.6359 & Sphere wall & 1 \\
4 & 0.8294 & Cylinder sloshing & 1 \\
5 & 0.9635 & Sphere sloshing & 0 \\
22 & 5.0785 & Cylinder wall & 0 \\
\hline
\end{tabular}

4.4 Modes and frequencies of the fluid-filled cylindrical-spherical tank

To define coupled modes of harmonic vibrations of the elastic tank, we represent the timedependant unknown coefficients in series for potentials $\Phi_{1}, \Phi_{2}$, eqn (11), and for vibration modes $\mathbf{u}$ of the fluid-filled shell, eqn (5), in the form

$$
c_{k}(t)=C_{k} e^{i \omega t} ; \quad d_{k}(t)=D_{k} e^{i \omega t},
$$

where $\omega$ is an own frequency, $C_{k}$ and $D_{k}$ are unknown constants. Using (14) we obtain the eigenvalue problem for evaluating natural frequencies and modes of the fluid-filled cylindrical-spherical tank [9].

The filling levels are $h_{1}=1 \mathrm{~m}$ and $h_{2}=1.5 \mathrm{~m}$. The lowest frequencies of the double tank are presented in Table 4.

The first frequencies are very close in value. For example, modes $1-5$ have frequencies with difference within $1 \mathrm{~Hz}$, and these modes are associated with different vibration types. The first twenty modes are predominantly sloshing ones.

\section{CONCLUSIONS}

The numerical procedure based on the coupling finite and boundary element methods is developed for the fluid-structure interaction analysis of the dual compartment tank. The considered problem has been solved using the multi-domain and single-domain boundary element approaches. The analysis demonstrates that sloshing and shell vibrations can not be considered separately. The lower frequencies of the fluid-filled tanks decrease to $0.2-0.4$ and $0.1-0.6$ of those of empty tanks for spherical and cylindrical compartments, respectively. The fluid-stricture interaction effects are more significant for the outer cylindrical part.

\section{ACKNOWLEDGMENTS}

The authors gratefully acknowledge Professor Carlos Brebbia, Wessex Institute of Technology, for his constant support and interest to our research.

\section{REFERENCES}

[1] Brebbia, C.A, Telles, J.C.F. \& Wrobel, L.C., Boundary Element Techniques: Theory and Applications in Engineering. Springer-Verlag: Berlin and New York, 1984.

[2] Wang, J. \& Gao, X.W., Structural multi-scale boundary element method based on element subdivision technique. Chinese Journal of Computational Mechanics, 27(2), pp. 258-263, 2010. 
[3] Biswal, K.C., Bhattacharyya, S.K. \& Sinha, P.K., Dynamic characteristics of liquid filled rectangular tank with baffles. IE (I) Journal-CV, 84, pp. 145-148, 2004.

[4] Kumar, A. \& Sinhamahapatra K.P., Dynamics of rectangular tank with perforated vertical baffle. Ocean Engineering, 126(1), pp. 384-401, 2016.

https://doi.org/10.1016/j.oceaneng.2016.09.012

[5] Faltinsen, O.M. \& Timokha, A.N., Sloshing. Cambridge University Press, Cambridge, 2009.

[6] Curadelli, O., Ambrosini, D., Mirasso, A. \& Amani, M., Resonant frequencies in an elevated spherical container partially filled with water: FEM and measurement. Journal of Fluids and Structures, 26(1), pp. 148-159, 2010.

https://doi.org/10.1016/j.jfluidstructs.2009.10.002

[7] Ravnik, J., Strelnikova, E., Gnitko V., Degtyarev, K. \& Ogorodnyk,U., BEM and FEM analysis of fluid-structure interaction in a double tank. Engineering Analysis with Boundary Elements, 67, pp. 13-25, 2016. https://doi.org/10.1016/j.enganabound.2016.02.006

[8] Wan, F.Y.M. \& Weinitschke, H.J., On shells of revolution with Love-Kirchoff hypothesis. Journal of Engineering Mathematics, 22, pp. 285-334, 1988. https://doi.org/10.1007/bf00058512

[9] Degtyarev, K., Glushich, P., Gnitko, V. \& Strelnikova, E., Numerical simulation of free liquid-induced vibrations in elastic shells. International Journal of Modern Physics and Applications, 1(4), pp. 159-168, 2015.

[10] Gnitko, V., Naumenko, V., Rozova, L. \& Strelnikova, E., Multi-domain boundary element method for liquid sloshing analysis of tanks with baffles. Journal of Basic and Applied Research International, 17(1), pp. 75-87, 2016.

[11] Gnitko, V., Degtyariov, K., Naumenko, V. \& Strelnikova, E., BEM and FEM analysis of the fluid-structure Interaction in tanks with baffles. International Journal of Computational Methods and Experimental Measurements, 5(3), pp. 317-328, 2017. https://doi.org/10.2495/cmem-v5-n3-317-328

[12] McIver, P., Sloshing frequencies for cylindrical and spherical containers filled to an arbitrary depth. Journal of Fluid Mechanics, 201, pp. 243, 1989.

https://doi.org/10.1017/s0022112089000923

[13] Faltinsen, O.M. \& Timokha, A.N., Analytically approximate natural sloshing modes for a spherical tank shape. Journal of Fluid Mechanics, 703, pp. 391-401, 2012. https://doi.org/10.1017/jfm.2012.237

[14] Kulczycki, T., Kwaśnicki, M. \& Siudeja, B., The shape of the fundamental sloshing mode in axisymmetric containers. Journal of Engineering Mathematics, 99(1), pp. 157-193, 2016.

https://doi.org/10.1007/s10665-015-9826-6 